\title{
Scaling Limit for the Space-Time Covariance of the Stationary Totally Asymmetric Simple Exclusion Process
}

\section{Patrik L. Ferrari, Herbert Spohn}

Zentrum Mathematik and Physik Department, Technische Universität München, Boltzmannstr. 3, 85747 Garching, Germany. E-mail: ferrari@ma.tum.de, spohn@ma.tum.de

Received: 17 March 2006 / Accepted: 17 March 2006

Erratum published online: 29 April 2006 - (C) Springer-Verlag 2006

Commun. Math. Phys. (2006) DOI 10.1007/s00220-006-1549-0

Unfortunately, Figs. 1, 3, and 4 were printed incorrectly, due to a publisher's error. The correct versions are shown below:

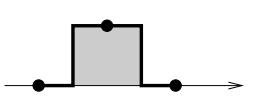

$\tau=1$

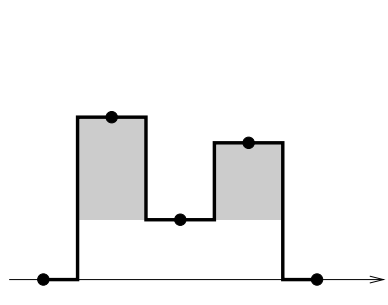

$\tau=2$

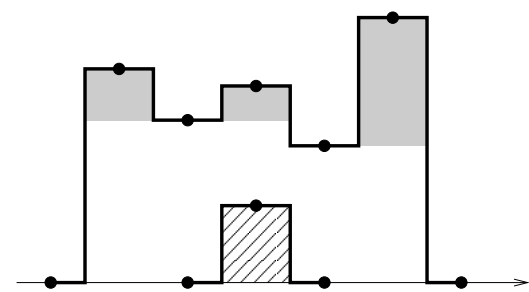

$\tau=3$

Fig. 1. The growth dynamics associated with the TASEP directed last passage percolation 


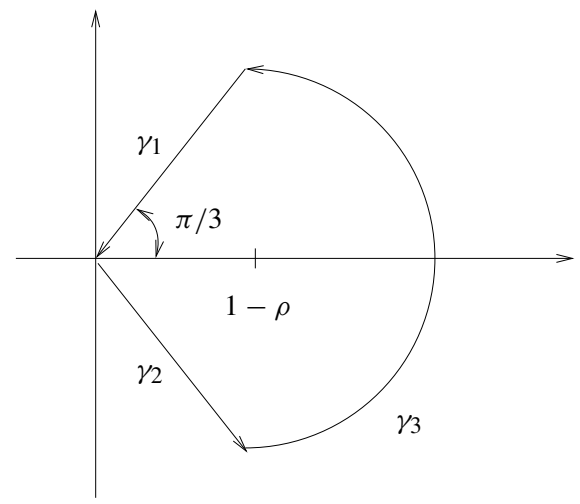

Fig. 3. Integration path used for the asymptotics for fixed $y$

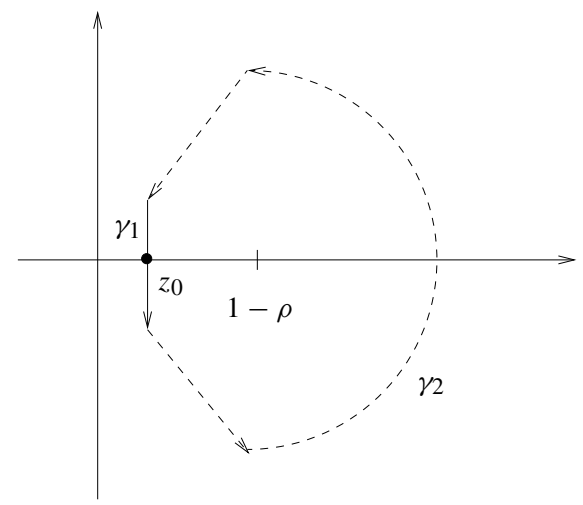

Fig. 4. Integration path used for the asymptotics for large value of $y . \gamma_{2}$ is the dashed line 